\title{
Enseñanza de metodologías de la investigación cientí- fica en la Universidad Nacional de Rosario (UNR) Una mirada sobre los planes de estudios El caso de Psicología
}

\author{
Andrea Espinosa ${ }^{1}$ \\ andrea_espinosa01@yahoo.com.ar \\ Rec. 30/02/2018, Apr. 14/04/2018
}

\begin{abstract}
Resumen
En este escrito se abordan algunas de las características sobresalientes respecto de la enseñanza de Metodología de la Investigación Científica en carreras de grado universitario en el contexto de la UNR. Una mirada inicial sobre la narrativa de los Planes de Estudios se realiza tomando el caso de Psicología como modelo dentro de las Ciencias Sociales. A partir del análisis de cuatro dimensiones básicas observadas en los documentos, así como en lo narrado por un docente, puede observarse la complejidad del entretejido de factores que influyen sobre la forma de pensar la enseñanza de contenidos metodológicos en este contexto.
\end{abstract}

Palabras claves: Enseñanza - Metodología - Universidad.

\begin{abstract}
This paper deals with some of the major characteristics of the teaching of Scientific Research Methodology in university degree careers at UNR. An initial look at the narrative of the Curriculum is done, taking the one from Psychology as a model from Social Sciences. From the analysis of four basic dimensions observed in the documents and the words from a teacher it can be understand the complexity of the complexity of the interweaving of factors that influence the way of thinking the teaching of methodological contents in this context.
\end{abstract}

Key words: Teaching - Methodology - University.

\footnotetext{
1 Psicóloga y Profesora en Psicología (UNR). Especialista en Metodología de la Investigación Científica (UNLa). Docente e Investigadora en Facultad de Psicología - Universidad Nacional de Rosario - Jefe de Trabajos Prácticos en Metodologías de la Investigación en Psicología (UNR). Docente de Metodología de la Investigación: Diseños Cuantitativos (Lic. en Psicología - UAI)
} 


\section{Introducción}

La enseñanza de metodologias de investigación cientifica se vincula con la introducción al conocimiento científico, su génesis, sus avances y sus transformaciones. Desde este punto de vista es el primer ámbito en el que la actividad científica tiene efectos al "[...] transformar las mentes de las personas y estructurarlas conforme a los contenidos, reglas metodológicas y valores que caracterizan a la ciencia”, como plantea Echeverría (2001, pp. 43-44). Las acciones educativas específicas relacionadas con la actividad científica están regidas por valores que deben ser satisfechos para que dichas acciones puedan ser consideradas científicas. Para ello, el autor establece que es necesario desarrollar un contexto de educación que sirva de marco para analizar esos valores. Sumado a ello, plantea que "[...] el aprendizaje es una condición sine qua non para la acción científica" (p.43) dado que la comprensión de un enunciado científico debe estar mediada por el aprendizaje de conocimientos complejos a través de los cuales, también, se aprende sobre los procedimientos científicos. Desde esta mirada puede puntualizarse que, enseñar Metodologías de la Investigación Científica en el grado universitario es un desafío que debiera ser estudiado desde una perspectiva multidimensional. Así abordado pueden visualizarse dimensiones que atañen a docentes y estudiantes, contenidos teóricos y prácticos, conocimientos adquiridos y construidos, modalidades de enseñanza, tiempos, marcos epistemológicos, entre muchas otras.

En este marco, las preguntas de las que se parte en esta investigación están encuadradas en la intención de trazar un recorrido desde distintas perspectivas respecto de los primeros pasos de la enseñanza de Metodologías de la Investigación Científica en carreras de grado universitario. Los interrogantes principales circunscriben al objeto de estudio en tanto se pregunta: ¿cómo se enseña metodologías de investigación científica en la universidad? Específicamente, ¿cómo piensan y analizan la enseñanza de este campo de saberes los docentes que tienen la función de iniciar a los estudiantes universitarios en temas científicos? Es así que este estudio intenta caracterizar la enseñanza de metodologías de investigación científica en las carreras de grado de la UNR desde la perspectiva de los programas de contenidos de las mismas.

Una tarea preliminar de este estudio se asienta en la lectura y análisis de los Planes de Estudios de las carreras de grado universitario de la UNR. El foco se dirige hacia el espacio narrativo de estos documentos, específicamente la Fundamentación de la carrera. Esta lectura permite identificar aquellas formaciones profesionales en las que aparece la investigación como uno de los aspectos en los que se formarán los estudiantes. En este caso, se tomará como eje de análisis el discurso escrito de las carreras, en la fundamentación de los Planes de aquellas formaciones profesionales que indiquen en su malla curricular la existencia de espacios denominados Metodología de la Investigación.

La hipótesis que guía este estudio refiere que la diversidad de metodologías de investigación científica así como las diferencias en las carreras de grado de la UNR miradas desde aspectos tales como la amplitud de la bibliografía, la duración del cursado, el lugar de la materia en el plan de estudios, la cantidad de estudiantes en el aula, las trayectorias docentes y la historia de la materia en el dictado de la carrera influyen sobre las características del inicio de la enseñanza de metodologías de investigación científica.

Las unidades de análisis con las que se trabaja en esta investigación son los documentos denominados de Planes de Estudios de las carreras de la UNR focalizando en aquellos que contengan materias denominadas Metodología de la Investigación. Las 
variables a observar serán las características asociadas a la producción de conocimiento científico detectadas.

La modalidad del estudio es descriptiva. Definida por Ynoub (2007) como aquella modalidad que destaca un tipo de investigaciones que se orientan a describir el comportamiento de las variables, identificar tipos o pautas características resultantes de combinaciones de valores entre varias de ellas.

Se considera que una perspectiva de análisis, asociado a perspectivas o enfoques cualitativo-inductivistas, de los planes de estudios es una fuente de información no sólo importante, sino necesaria, para la comprensión del estatus que se le da en las diferentes áreas de conocimiento a la alfabetización científica de los profesionales en formación. Este recorrido forma parte del camino que puede contribuir al diálogo en la superación de algunos obstáculos comunes que tienen quienes se dedican a la enseñanza de metodologías de investigación cientifica en las carreras de grado universitario de la UNR.

Como caso modelo, para el desarrollo de este escrito, se toma la carrera de Psicología. En este caso particular, debido a las exigencias planteadas por la pertenencia de esta profesión al artículo 43 de la Ley de Educación Superior No 24.521 (LES), es decir que corresponde a las carreras consideradas de interés público, y a la necesidad de acreditación de la misma planteada por la Comisión Nacional de Evaluación y Acreditación Universitaria (CoNEAU), se realizó una modificación del Plan de Estudios en el año 2014. Por esta razón se toma para el análisis el nuevo documento, aprobado por Resolución 140/14 CD.

\section{Desarrollo}

Se parte de la concepción que enseñar Metodologías de la Investigación Científica en el grado universitario es un desafío que debe ser estudiado desde una perspectiva multidimensional. Así abordado pueden visualizarse dimensiones que atañen a docentes y estudiantes, contenidos teóricos y prácticos, conocimientos adquiridos y construidos, modalidades de enseñanza, tiempos, marcos epistemológicos, entre muchos otras.

Las categorías en estudio que se observarán serán: investigación, metodología de la investigación, formación científica, perfil profesional. Para realizar el análisis de estas categorías se recurre a la construcción de una matriz que permite observar las categorías y sus interacciones, a partir de lo hallado en dos apartados bien definidos en la Fundamentación de los Planes de Estudios: Finalidad del Plan de Estudios y Perfil del Título.

La modalidad de abordaje de los documentos da cuenta de un enfoque metodológico con características inductivistas, como se menciona más arriba. Subyace de esta manera una intencionalidad, la de trabajar en la comprensión de las categorías, su uso y contextualización.

En un segundo momento, se aborda el Plan de Estudios como la Unidad de Análisis perteneciente al Nivel de Anclaje Base, cuyas variables serán la Fundamentación y el Perfil del Título. En este sentido, se entiende que la Unidad de Análisis del Supra-Nivel es la Universidad; en tanto que, en el Sub-Nivel la Unidad de Análisis es la Metodología de la Investigación.

La selección, para este trabajo, de la carrera de Psicología como caso en el estudio se sostiene sobre dos aspectos contextuales diferentes que confluyen en una mirada más abarcativa y profunda. El primero de ellos, el contexto externo, reviste la mirada sobre el nuevo documento, producto de la necesidad de responder a los requerimien- 
tos establecidos en los estándares para la acreditación de carreras de interés público (enmarcadas en el art. 43 de la LES) y ejecutada por los evaluadores de la CoNEAU. El documento sometido a análisis es el que se encuentra vigente, en este marco, de acuerdo a la Resolución 140/14 CD. En segundo término, siendo miembro de la planta docente de esta casa de altos estudios desde hace dos décadas (asociado al contexto interno), es de destacar la participación desde el claustro docente, con distintos grados de implicancias, en la historia académica y política de la institución. Este segundo contexto brinda un conocimiento de detalles, recorridos e intersticios que en el documento definitivo han quedado velados.

Respecto de los docentes responsables de las materias denominadas Metodología de la Investigación, se trabajará con los profesores titulares y los profesores de mayor antigüedad de los espacios curriculares que se hallen en primer o segundo año de la malla curricular de una carrera paradigmática de cada subgrupo (ciencias naturales y ciencias sociales), de acuerdo a lo establecido más arriba. Los titulares de cátedra tienen una visión integral de la enseñanza de la materia en la que trabajan. Las personas con mayor antigüedad suelen tener críticas a las transformaciones históricas que el programa ha tenido, aportando una visión también integral a las características de la enseñanza de su materia.

\section{Abordaje metodológico}

En la búsqueda de las categorías de análisis propuestas (Investigación - Metodología de la Investigación - Formación Científica - Perfil Profesional asociado a Investigación) se recorre el texto observando la aparición específica de los términos. Luego, se construye una matriz a partir de la cual pueden hacerse interpretaciones que insten, en un tercer paso, a la comprensión a la luz del contexto socio-histórico-político de los resultados.

Sólo se transcriben en la Tabla las oraciones en las que se observa claramente la aparición de los términos previamente establecidos como categorías de análisis. Se dejan de lado, en este momento, las ideas que de forma indirecta o velada den cuenta de las categorías entendiendo que están mediadas por los conocimientos previos del investigador. Se decidió dejar este paso para un momento posterior.

Se trabaja con los docentes a partir de entrevistas abiertas a través de cuyas narraciones se busca identificar las categorías en estudio, sus definiciones y su contextualización histórico-política y social.

\section{Análisis Preliminar de Resultados}

En el abordaje de los documentos lo que puede leerse, a simple vista, es la ausencia de la denominación Metodología de la Investigación en ambos apartados. En el Perfil del Título sólo se hace referencia, en forma directa, a la formación científica. En todos los casos, la referencia que se hace a las categorías en estudio se apoya en la idea de análisis o utilización de conocimientos, tanto teóricos como empíricos (en términos de herramientas de medición o de intervención clínica). Sin embargo, la idea de producción de conocimiento, como partícipes o artífices de ello, aparece con escasa fuerza. 
Andrea Espinosa / Enseñanza de metodologías de la investigación [...] /139

\begin{tabular}{|c|c|c|}
\hline & Fundamentación & Perfil del Título \\
\hline Investigación & $\begin{array}{l}\text { Investigación de los procesos } \\
\text { psicológicos de los sujetos }\end{array}$ & \\
\hline \multicolumn{3}{|l|}{$\begin{array}{l}\text { Metodología de la } \\
\text { Investigación }\end{array}$} \\
\hline Formación Científica & $\begin{array}{l}\text { Tiene competencias para utilizar } \\
\text { los conocimientos científicos y } \\
\text { técnicos en la resolución de pro- } \\
\text { blemas relativos a los conflictos } \\
\text { psicológicos }\end{array}$ & $\begin{array}{l}\text { profesionales capacitados } \\
\text { científicamente, ubicados his- } \\
\text { tórica y epistemológicamente }\end{array}$ \\
\hline Perfil Profesional & $\begin{array}{l}\text { Posee la capacidad necesaria } \\
\text { para aplicar las metodologías de } \\
\text { investigación propias del estudio } \\
\text { y análisis de problemáticas en } \\
\text { sus campos de intervención. }\end{array}$ & \\
\hline
\end{tabular}

Tabla 1

Las características narrativas de los apartados del Plan de Estudios muestra una fuerte impronta profesionalista, con un marcado acento teórico, que identifica a las carreras de ciencias sociales prácticamente desde sus inicios.

El Plan de Estudios de la carrera de Psicología, tomada como modelo paradigmático de las Ciencias Sociales, en su última modificación en el año 2014, muestra una estructura en la que se destaca el desarrollo narrativo de cada una de las instancias identificadas. El desarrollo más extensivo se destaca, justamente, en los apartados seleccionados para el análisis: la fundamentación del plan de estudios y el perfil del título. Hay que destacar que en este segundo ítem se vuelcan fragmentos de la Ley de Ejercicio Profesional del Psicólogo y Licenciado en Psicología (Ley Provincial 9538), detallando las Incumbencias o Actividades Reservadas al Título. Dado que la propuesta de este estudio está vinculada con la observación de la formación de grado en temas de producción científica, se decidió desestimar esta información.

En el discurso de los docentes surge fuertemente la necesidad de contextualizar las carreras y sus Planes, relacionando sus modificaciones y sus rasgos epistemológicos subyacentes con los modelos sociales y científicos preponderantes en cada momento histórico.

La perspectiva de los docentes será, a su vez, procesada a través de una matriz con categorías preestablecidas (coincidentes con las descriptas para el análisis de los documentos) y analizada por medio de la técnica de análisis de contenido.

Los contextos, externo e interno, proveen de datos complementarios que permiten una comprensión mayor sobre lo acontecido en el análisis preliminar de las categorías en estudio.

En primera instancia - desde un contexto externo -, podría pensarse que en función de los hallazgos la noción de formación científica en términos de producción de conocimientos se encuentra ausente de la currícula de la carrera de Psicología, que ha sido tomada como modelo paradigmático dentro del subgrupo de las Ciencias Sociales. Sin embargo, una lectura pormenorizada puede indicar que subyacen en el relato indicios que presentan una visión ampliada en tanto usuarios y productores de conocimientos científicos en Psicología. 
Es probable que la falta de indicadores directos sobre estas categorías tengan su base en la historia de la carrera con su sesgo profesionalista clínico. Este dato no es menor dado que el modelo teórico a partir del que se trabaja, tanto la formación como la intervención clínica, es de base psicoanalítica. El posicionamiento epistemológico de este modelo se ha parado en sus inicios desde un enfoque hermenéutico (Freud lo ha planteado abiertamente en sus teorizaciones). Sin embargo, desde hace unas décadas esta postura ha ido virando hacia un modelo más extremo en el que se ha establecido que, para esta teoría, la investigación es "imposible" (modelo lacaniano).

Otro punto proveniente del contexto externo, la inminencia de la acreditación de la carrera por parte de la CoNEAU, impuso la necesidad de realizar modificaciones, en función de los estándares, al Plan de Estudios vigente hasta 2013, en tiempos y con exigencias diferentes a los de la institución. Esta "urgencia" puede tener correlato sobre lo escrito en el documento.

Desde el contexto interno, la impronta psicoanalítica lacaniana de la carrera (aunque se intenta suavizar) tiene una lectura sesgada de las implicancias de la producción de conocimientos, sus formas y su necesidad. Este sesgo produce una situación tirante, carente de debate en algunos ámbitos, que ha producido como efecto falta de claridad en algunas narraciones del documento analizado.

Estas perspectivas son primeros análisis o primeras lecturas e interpretaciones de la búsqueda de las categorías de análisis en el Plan de Estudios de la carrera. La intención es, una vez trabajado con mayor profundidad el documento, trasladar esta misma forma de análisis a un Plan de Estudios de una carrera representativa de las Ciencias Naturales.

\section{Discusión}

Como primer abordaje de los documentos estudiados, tomando como modelo del subgrupo de las Ciencias Sociales la carrera de Psicología, y la entrevista a un profesor de la primera materia denominada Metodología de la Investigación de la misma carrera, se observa que existen indicios que permiten acompañar las vinculaciones que se realizan en la hipótesis entre las diferentes dimensiones involucradas en el estudio.

La articulación de los contenidos hallados en el Plan de Estudios con los decires del docente muestran una mirada contextual necesaria para comprender la ubicación de la materia en la malla curricular, así como la mirada social y política direcciona los contenidos que se trabajan con los estudiantes a la hora de introducirlos a la reflexión sobre la vida científica de una disciplina tan compleja como es la Psicología.

En cuanto a la aplicación de las herramientas metodológicas es importante el posicionamiento desde un tipo de estudio particular para poder conocer la manera en que ese objeto de estudio será interpretado y comprendido desde los diferentes interrogantes postulados como preguntas de investigación. Dicho objeto no puede concebirse fuera de una matriz de datos, ya que lo contempla, incorpora y contiene como unidad de análisis.

Finalmente, una limitación inicial de esta discusión es su carácter de preliminares o iniciales, dado que hasta el momento sólo se ha trabajado con un sub-grupo de ciencias, teniendo en consideración el agrupamiento de las carreras. Este sesgo da cuenta de los vínculos mostrados en la hipótesis. Sin embargo, habrá que trabajar analíticamente sobre una carrera que pueda ser considerada paradigmática dentro del agrupamiento de las Ciencias Naturales. 


\section{Bibliografía}

- Ambrosini, C. y Beraldi, G. (2015). Pensar la ciencia hoy. La epistemología: entre teorías, modelos y valores. Buenos Aires. Educando.

- Echeverría, J. (2001). Enseñanza de la ciencia y educación en valores. ÉNDOXA: Series Fisolóficas. UNED, Madrid. (14), 41-59

- Ley de Ejercicio Profesional del Psicólogo y Licenciado en Psicología de la Provincia de Santa Fe N ${ }^{\circ}$ 9.538. Recuperado de: http://fepra.org.ar/feprav3/documentos/ leyes_ejercicio_provincias/Santa-Fe_Ley-9538.pdf

— Ley de Educación Superior No 24.521. Recuperado de: http://www.me.gov.ar/consejo/cf_leysuperior.html

- Plan de Estudios de la Carrera de Psicología de la Universidad Nacional de Rosario. Resolución de CD 140/14. Recuperado de: http://www.fpsico.unr.edu.ar/?page_id $=741$

- Ynoub, R. (2015). Cuestión de Método. Aportes para una metodología crítica. Cengage Learning. México. 